\title{
Spin Hall and Edelstein Effects in Metallic Films
}

\begin{abstract}
J.Borge ${ }^{a, *}$, C. GORIni ${ }^{b}$, G. Vignale ${ }^{c}$ And R. RAimondi ${ }^{a}$
${ }^{a}$ Dipartimento di Matematica e Fisica, Università Roma Tre, Via della Vasca Navale 84, Rome, Italy ${ }^{b}$ Service de Physique de l'État Condensé, CNRS URA 2464, CEA Saclay, F-91191 Gif-sur-Yvette, France

${ }^{c}$ Department of Physics and Astronomy, University of Missouri, Columbia MO 65211, USA

In an insulator-metal-insulator junction, inversion-symmetry breaking at the interfaces between the 3D metallic film and the top and bottom insulating layers may give rise to a sizeable (Rashba-like) spin-orbit interaction. In this paper we study the spin Hall and Edelstein effects produced by such an interface interaction through a quasiclassical approach. We find that the spin Hall conductivity has a finite value even if spin-orbit interaction with impurities in the bulk of the metallic film is neglected and disorder is properly taken into account. This is in sharp contrast with the case of a strictly 2D metallic layer, in which case impurity scattering is known to completely suppress Rashba-like contributions to the spin Hall conductivity. The non-vanishing of the latter has a profound influence on the Edelstein effect, which we show to consist of two terms, the first with the standard form valid in an exactly $2 \mathrm{D}$ system, and a second arising from the presence of the third dimension.
\end{abstract}

DOI: $10.12693 /$ APhysPolA.127.457

PACS: 72.25.Mk, 75.70.Tj, 85.75.-d

\section{Introduction}

In the last two decades spintronics has become a central topic in condensed matter physics. In this context spin-orbit coupling (SOC) has attracted a lot of attention, since it gives rise to numerous interesting transport effects. Among them, the spin Hall effect (SHE) and Edelstein effect (EE) stand out not the least because of their potential technological importance. The spin Hall effect consists in the appearance of a $z$-polarized spin current flowing in the $y$-direction produced by an electric field in the $x$-direction. The Edelstein effect [1] consists instead in the appearance of a $y$-spin polarization in response to an applied electric field in the $x$-direction.

It is a well known result that the Spin Hall Conductivity (SHC) vanishes in the $2 \mathrm{D}$ Rashba model. However it was recently pointed out that such vanishing does not occur in models that are not strictly two-dimensional [2]. Furthermore, a new model in which a thin (but 3D) metallic film without any bulk SOC is sandwiched between two different insulators lead to a large spin-Hall angle $[3,4]$, and to a substantial modification of the standard Edelstein effect as well [4]. These phenomena can be traced back to the Rashba-like SOC appearing at the top and bottom metal-insulator contacts, where inversion symmetry across the interfaces is broken. Thus, metals without substantial bulk spin-orbit can yet host sizeable spin-orbit induced effects.

In this paper we will study the spin Hall and Edelstein effects in a insulator-metal-insulator junction by means of the quasiclassical Eilenberger equation [5]. This approach has proven highly effective in revealing the hidden and non-trivial relationship existing between the spin

*corresponding author; e-mail: borge@fis.uniroma3.it
Hall and Edelstein effects [5-7]. This relationship has been further explored [4, 8], and we will see here that it is not limited to $2 \mathrm{D}$ systems, but can be suitably generalized to the presently explored 3D, asymmetrically confined metallic film. The relationship itself can be formulated by writing the Edelstein effect conductivity $\sigma^{\mathrm{EE}}$ as

$$
\sigma^{\mathrm{EE}}=\frac{\tau_{s}}{L_{s o}}\left(\sigma^{\mathrm{SHE}}-\sigma_{\mathrm{drift}}^{\mathrm{SHE}}\right),
$$

where $\sigma_{\text {drift }}^{\mathrm{SHE}}=e \tau /\left(4 \pi \hbar \tau_{s}\right)$ is the direct ("drift") spin Hall conductivity generated by the driving electric field, whereas $\sigma^{\mathrm{SHE}}$ is the full one, containing diffusion contribution as well [4]. In the above, $\tau$ is the momentum scattering time, $\tau_{s}$ the spin relaxation time and $L_{s o}$ the spin relaxation length.

It is known that in the Rashba model a general constraint from the equation of motions leads to $\sigma^{\mathrm{SHE}}=0$, and the Edelstein Conductivity (EC) can be written as $\sigma^{\mathrm{EE}}=-e N_{0} \alpha \tau$. However in a 3D system with inhomogeneous SOC such a constraint is absent [2], and indeed we will find that in our metallic thin film a non zero SHC exists, with important consequences for EC.

\section{The model}

Following Refs. [3, 4], we model the normal metallic thin film via the following Hamiltonian

$$
H=\frac{p^{2}}{2 m}+V_{C}(z)+H_{R}+U(\boldsymbol{r}),
$$

where the first term represents the kinetic energy. The finite thickness $d$ of the metallic film is taken into account by a confining potential

$$
V_{C}=V_{+} \theta\left(z-z_{+}\right)+V_{-} \theta\left(z_{-}-z\right),
$$

where $V_{ \pm}$is the height of the potential barrier at $z_{ \pm}=$ $\pm d / 2$ and $\theta(z)$ is the Heaviside function. The Rashba interfacial spin-orbit interaction in the $x-y$ plane located at $z_{ \pm}= \pm d / 2$ is described by 


$$
\begin{aligned}
& H_{R}= \\
& \frac{\lambda_{-}^{2} V_{-} \delta\left(z-z_{-}\right)-\lambda_{+}^{2} V_{+} \delta\left(z-z_{+}\right)}{\hbar}\left(p_{y} \sigma_{x}-p_{x} \sigma_{y}\right),
\end{aligned}
$$

where $\lambda_{ \pm}$are the effective Compton wavelengths for the two interfaces and $\sigma_{x}, \sigma_{y}, \sigma_{z}$ the Pauli matrices. The last term in Eq. (2) is the white-noise impurity potential, taken with variance $\left\langle U(\boldsymbol{r}) U\left(\boldsymbol{r}^{\prime}\right)\right\rangle=\left(2 \pi N_{0} \tau\right)^{-1} \delta\left(\boldsymbol{r}-\boldsymbol{r}^{\prime}\right)$, where $N_{0}=m / 2 \pi \hbar^{2}$ is the two-dimensional density of states and $\boldsymbol{r}=(x, y)$.

The Hamiltonian (2) (without disorder) can be diagonalized, though an explicit form for its eigenvalues can only be obtained within perturbation theory - notice that in the absence of interfacial spin-orbit such eigenvalues reduce to the kinetic energy plus the infinite potential well levels $E_{0} n^{2}=\hbar^{2} \pi^{2} n^{2} /\left(2 m d^{2}\right)$. There are two natural length scales associated with the confining potential $d_{ \pm}=\hbar / \sqrt{2 m V_{ \pm}}$, and we base our perturbative treatment on the assumption that $d_{ \pm} / d \ll 1$. We also define $\alpha_{ \pm}=\lambda_{ \pm}^{2} / d_{ \pm}$. We work in the following with natural units $(\hbar=1$ and $c=1)$, and make an expansion to first order in $d_{ \pm} / d$ and up to third order in $\alpha_{ \pm} p$. Note that $E_{0} \alpha_{ \pm}$has the dimensions of a velocity, suggesting an analogy with the typical Rashba coupling parameter $\alpha$ of $2 \mathrm{D}$ systems. The eigenvalues of the Hamiltonian are

$$
\begin{aligned}
& \epsilon_{n \boldsymbol{p} s}= \\
& \quad E_{0} n^{2}\left[1-2 \frac{d_{-}+d_{+}}{d}+s e_{1} p+e_{2} p^{2}+s e_{3} p^{3}\right],
\end{aligned}
$$

where

$$
\begin{aligned}
e_{i} & =2(-1)^{i+1}\left(\frac{d_{+}}{d} \alpha_{+}^{i}+(-1)^{i} \frac{d_{-}}{d} \alpha_{-}^{i}\right), \\
i & =1,2,3 .
\end{aligned}
$$

The eigenfunctions as well will depend on $z$, but this dependence is not crucial for our present purpose and will not be considered. We will also neglect inter-band effects, which is appropriate as long as the inter-band spacing $E_{0}$ is larger than the intra-band spin-orbit splitting or the disorder broadening [4].

\section{The quasiclassical approach}

It is useful to adopt an heuristic point of view and develop the quasiclassical approach building on the analogy with the well known 2D Rashba case. To this end we begin by recalling the Eilenberger equation of motion for the quasiclassical Green function $\check{g}$, which is a matrix in Keldysh space. Following Eq. (13) of Ref. [5], ̌̆ obeys the equation

$$
\begin{aligned}
& \sum_{s= \pm}\left(\partial_{t} \check{g}_{s}+\frac{1}{2}\left\{\frac{\boldsymbol{p}_{s}}{m}+\frac{\partial}{\partial \boldsymbol{p}}\left(\boldsymbol{b}_{s} \cdot \boldsymbol{\sigma}\right), \frac{\partial}{\partial \boldsymbol{x}} \check{g}_{s}+\mathrm{i}\left[\boldsymbol{b}_{s} \cdot \boldsymbol{\sigma}, \check{g}_{s}\right]\right\}\right)= \\
& -\mathrm{i}[\check{\Sigma}, \check{g}],
\end{aligned}
$$

where, in the $2 \mathrm{D}$ Rashba case, $\boldsymbol{b}_{s}=\alpha\left(\boldsymbol{p}_{s} \times \boldsymbol{e}_{z}\right)$ is the momentum dependent SOC and $\check{\Sigma}$ is the self-energy matrix in the Born approximation. Later on we will replace $\boldsymbol{b}$ with the appropriate expression for the model discussed in the previous section. The retarded component $g^{R}$ (and similarly the advanced one $g^{A}$ ) is written as a sum of the contributions associated to the two spin-split Fermi surfaces $g_{ \pm}^{R}=\left(1 \mp \partial_{\xi} b\right)\left(\frac{1}{2} \pm \frac{1}{2} \hat{\boldsymbol{b}} \cdot \boldsymbol{\sigma}\right)$. The electrical field is normally included in the quasiclassical equations of motion by substituting $\partial_{\boldsymbol{x}} \rightarrow \partial_{\boldsymbol{x}}-|e| \boldsymbol{E} \partial_{\epsilon}$. In the absence of the field the equilibrium Keldysh component is $g=2 \tanh \left(\frac{\xi}{2 T}\right) g^{R}$. By representing the matrices in the eigenstates basis [6], the Keldysh component of Eq. (7) reads

$$
\begin{aligned}
& \hat{p}_{x} E\left(v_{F}+\sigma_{z} \frac{p_{+} N_{+}-p_{-} N_{-}}{2 N_{0} m}\right)+\left(\sigma_{z} \hat{p}_{x}-\sigma_{y} \hat{p}_{y}\right) \alpha E \\
& \quad+\mathrm{i} \alpha p\left[\sigma_{z}, g\right]=-\frac{1}{\tau}(g-\langle g\rangle),
\end{aligned}
$$

where $g$ has still a structure in spin space $g=g_{0} \sigma_{0}+$ $\sum_{i} g_{i} \sigma_{i}$ and $\langle g\rangle_{0}=\left\langle g_{0}\right\rangle,\langle g\rangle_{x}=\left\langle g_{x}\right\rangle$, with

$$
\begin{aligned}
& \langle g\rangle_{y}=\hat{\boldsymbol{p}} \cdot\left\langle\hat{\boldsymbol{p}} g_{y}\right\rangle+\hat{\boldsymbol{p}} \times\left\langle\hat{\boldsymbol{p}} g_{z}\right\rangle \\
& \langle g\rangle_{z}=-\hat{\boldsymbol{p}} \times\left\langle\hat{\boldsymbol{p}} g_{y}\right\rangle+\hat{\boldsymbol{p}} \cdot\left\langle\hat{\boldsymbol{p}} g_{z}\right\rangle,
\end{aligned}
$$

where langle... is the angular average over $\hat{\boldsymbol{p}}$. In this basis the spin current, $j_{y}^{z}$, and the spin polarization, $s^{y}$, are expressed as

$$
\begin{aligned}
j_{y}^{z} & =\frac{N_{0}}{4} \int d \epsilon\left\langle v_{y} g_{x}\right\rangle, \\
s^{y} & =-\frac{N_{0}}{4} \int d \epsilon\left(\left\langle\hat{p}_{y} g_{y}\right\rangle-\left\langle\hat{p}_{x} g_{z}\right\rangle\right) .
\end{aligned}
$$

After projecting Eq. (8) on the Pauli matrix components and solving the equations one finds

$$
\begin{aligned}
&\left\langle\hat{p}_{y} g_{x}\right\rangle=-\frac{E}{4 b_{0}}\left(2 \alpha+\frac{p_{+} N_{+}-p_{-} N_{-}}{2 N_{0} m}\right) \\
&\left\langle\hat{p}_{y} g_{y}\right\rangle=\frac{1}{2 b_{0} \tau}\left\langle\hat{p}_{y} g_{x}\right\rangle \\
&\left\langle\hat{p}_{x} g_{z}\right\rangle=-E \tau\left(\alpha+\frac{p_{+} N_{+}-p_{-} N_{-}}{2 N_{0} m}\right) \\
&-\frac{1}{2 b_{0} \tau}\left\langle\hat{p}_{y} g_{x}\right\rangle .
\end{aligned}
$$

In the Rashba case we have

$$
\frac{p_{+} N_{+}-p_{-} N_{-}}{2 N_{0} m}=-2 \alpha,
$$

yielding the well known cancellation of the SHC between the contribution from the bare bubble, $2 \alpha$, and the vertex corrections $\left(p_{+} N_{+}-p_{-} N_{-}\right) /\left(2 N_{0} m\right)$ (see also Eq. (B3) of Ref. [5]). The above form for the two contributions suggest how to straightforwardly extend the approach to our model. Hence, heuristically we make the following substitutions

$$
\begin{aligned}
& \alpha \rightarrow \frac{\epsilon_{n p+}-\epsilon_{n p-}}{2 p}=E_{0} n^{2}\left(e_{1}+e_{3} p_{F n}^{2}\right) \\
& \frac{p_{+} N_{+}-p_{-} N_{-}}{2 N_{0} m} \rightarrow E_{0} n^{2}\left(-2 e_{1}-4 e_{3} p_{F n}^{2}\right) .
\end{aligned}
$$

The above heuristic substitution can be proven rigorously by projecting Eq. (7) on each subband with eigenvalue $\epsilon_{n p s}$ and integrating over $\mathrm{z}$ the corresponding eigenfunctions. Full details of the derivation will be presented 
elsewhere. By inserting the substitutions (16)-(17) into (12)-(14) $j_{y}^{z}$ and $s^{y}$ read

$$
\begin{aligned}
j_{y}^{z} & =-\sum_{n}^{n_{c}} \frac{e}{4 \pi}\left(\frac{e_{3} p_{F n}^{2}}{e_{1}}\right) E \\
s^{y} & =-\sum_{n}^{n_{c}} e N_{0} \tau E_{0} n^{2} \\
& \left(e_{1}+3 e_{3} p_{F n}^{2}+\frac{2 e_{3}}{\left(2 \tau E_{0} n^{2}\left(e_{1}+e_{3} p_{F n}^{2}\right)\right)^{2}}\right) E,
\end{aligned}
$$

where $n_{c}$ is the number of occupied bands, and $p_{F n}^{2} / 2 m=\mu-E_{0} n^{2}$, the Fermi momenta for each subband. This is in agreement with the results obtained by a diagrammatic evaluation of the Kubo formula [4].

\section{Discussion of the results}

First of all we notice that both conductivities, Eqs. (18)-(19), are expressed as sums of single subbands contributions. By identifying $L_{s o}=p_{F n} / 2 m|\boldsymbol{b}|$ and $\tau_{s} / \tau=2\left(1+(2 \tau|\boldsymbol{b}|)^{2}\right) /(2 \tau|\boldsymbol{b}|)^{2}$, these quantities satisfy Eq. (1) for each subband. So with this result we should conclude that Eq. (1) is valid also in models with Rashba type spin-orbit coupling that are not strictly $2 \mathrm{D}$.

Another important conclusion from Eq. (1) is the appearance of an "anomalous" term in the EC due to the existence of a non-vanishing SHC.

There are two interesting physical situations that we are able to describe with this model. The first one consists on an insulator-metal-vacuum junction, where $\lambda_{+}=0, V_{+} \rightarrow \infty ; \lambda_{-}=\lambda, V_{-}=V$. In this case we get

$$
\begin{aligned}
\sigma^{\mathrm{EE}} & =-\sum_{n}^{n_{c}} \frac{2 e N_{0} \tau E_{0} n^{2} \lambda^{2}}{d}\left(1+\frac{\pi^{2} V}{8 \tau^{2} E_{0}^{3} n^{4}}\right) \\
\sigma^{\mathrm{SHE}} & =-\sum_{n}^{n_{c}} \frac{e}{4 \pi} 2 m p_{F n}^{2} V \lambda^{4} .
\end{aligned}
$$

There are some experimental studies of metal-metalvacuum junctions that show giant spin-orbit coupling [9]. If we have an insulator instead of a metal we expect better spin-transport coefficients (bigger gap, $V$ )

The second situation is the junction with the same spin-orbit coupling at the interfaces $\left(\lambda_{+}=\lambda_{-}\right.$or $\left.e_{1}=0\right)$, but different gap $\left(V_{-} \neq V_{+}\right)$. In this case we obtain a surprising result for both conductivities:

$$
\begin{aligned}
\sigma^{\mathrm{SHE}} & =-\sum_{n}^{n_{c}} \frac{e}{4 \pi} \\
\sigma^{\mathrm{EE}} & =\sum_{n}^{n_{c}} e N_{0} \tau \frac{2|\boldsymbol{b}|}{p_{F n}}\left(3+\frac{1}{2(2 \tau|\boldsymbol{b}|)^{2}}\right) .
\end{aligned}
$$

The result (22) for the SHC looks universal, i.e. does not depend on the strength of the SOC, as the one in the strictly 2D Rashba model [10] without disorder. However, this universality is not the result of ignoring vertex corrections, which are included in the quasiclassical approach, but rather of the independent subband approximation, which has its domain of validity. Indeed, the above result is not valid in the region where $V_{+} \rightarrow V_{-}$, where inter-band contributions become important.

\section{Conclusions}

In this paper we have calculated the Edelstein and spin Hall conductivities for a thin metallic film sandwiched by two different insulators. We have done it using the Eilenberger equations of motion. We have seen that the non-vanishing of the $\mathrm{SHC}$ is a direct consequence of the non strictly two-dimensional behavior of the spin-orbit coupling. This dependence on the $z$ component is the biggest difference with the $2 \mathrm{D}$ Rashba model. We have also proved that this non vanishing $\mathrm{SHC}$ gives rise to an anomalous term in the EC, which scales with the inverse of the scattering time and a "normal" one proportional to it. We have also proved the relation between the SHE and the EE if we move away from strictly two-dimensional spin-orbit interactions.

\section{References}

[1] V. Edelstein, Solid State Comm. 73, 233 (1990).

[2] L.X. Hayden, R. Raimondi, M.E. Flattè, and G. Vignale, Phys. Rev. B 88, 075405 (2013).

[3] X. Wang, J. Xiao, A. Manchon, and S. Maekawa, Phys. Rev. B 87, 081407 (2013).

[4] J. Borge, C. Gorini, G. Vignale, R. Raimondi, Phys. Rev. B 89, 245443 (2014).

[5] R. Raimondi, C. Gorini, P. Schwab, M. Dzierzawa, Phys. Rev. B 74, 035340 (2006).

[6] C. Gorini, P. Schwab, M. Dzierzawa, R. Raimondi, Phys. Rev. B 78, 125327 (2008).

[7] R. Raimondi, P. Schwab, C. Gorini, G. Vignale, Ann. Phys. (Berlin) 524, 153 (2012).

[8] cited, but omitted on the list!!!!K. Shen, G. Vignale, and R. Raimondi, Phys. Rev. Lett. 112, 096601 (2014).

[9] A.G. Rybkin, A.M. Shikin, V.K. Adamchuk, D. Marchenko, C. Biswas, A. Varykhalov, O. Rader, Phys. Rev. B 82, 233403 (2010).

[10] J. Sinova, D. Culcer, Q. Niu, N. A. Sinitsyn, T. Jungwirth, A. H. MacDonald, Phys. Rev. Lett. 92, 126603 (2004). 